\title{
A NOTE ON SETS OF OVERLAPPING INTERVALS.
}

\author{
By W. H. Young (Cambridge, Engl.).
}

Aduranza del 12 novembre 1905.

A new method of proving a set to be countable when each of its points is of countable order ${ }^{*}$ ), has lately been communicated by letter to my wife by Dr. Felix Bernstern; the proof is being published in the "Acta Mathematica ». This has suggested to me that the same method may be applied to prove a theorem with respect to sets of intervals given by me in a former paper ${ }^{* *}$ ). BERNSTErn's method consists in the application of the following simple Lemma and Corollary:

Lemma. - If $G$ be a countable set, any set of intervals whose end-points are points of $G$, is countable.

For, denoting the end-points by $P_{1}, P_{2}, \ldots$ and the interval whose end points are $P_{1}$ and $P_{2}$ by $(1,2)$, we may arrange these brackets in the customary form of a wedge,

$$
\begin{aligned}
& (\mathrm{I}, 2)(\mathrm{I}, 3)(\mathrm{I}, 4)(\mathrm{I}, \mathrm{S}) \ldots \\
& (2,3)(2,4)(2,5) \ldots \\
& (3,4)(3,5) \ldots \\
& (4,5) \ldots
\end{aligned}
$$

and " count " them in columns in order.

Corollary. - Any set of intervals whose end-points are rational is countable.

The following is the proof of my theorem above referred to.

THEOREM. - Given any set of intervals, overlapping in any manner, on a straight line, a countable set from among them may be found having the same internal points $\left.{ }^{* * *}\right)$.

If $P$ be any point of one of the intervals $(A, B)$, there is a rational point $R$ between $A$ and $P$, and one $R^{\prime}$ between $P$ and $B$. Thus there is an interval $\left(R, R^{\prime}\right)$, with rational end-points, containing $P$, and lying inside $(A, B)$.

Hence the intervals, each of which has rational end-points and is internal to one

*) That is to say an interval can be found containing the point and only a countable set of points of the given set.

$\rightarrow$ Overlapping Intervals [Proceed. London Mathem. Society, XXXV (Ig02-1903), p. 384].

***) An internal point of a set of intervals is a point internal to at least one of the intervals. 
of the given intervals, contain as internal points every point internal to the given intervals. By the preceding corollary these intervals are countable, and may therefore be denoted by

$$
\delta_{1}, \delta_{2}, \delta_{3}, \ldots
$$

Take an interval of the given set containing $\delta_{1}$, and denote it by $d_{1}$. Let the first of the $\delta$ 's not contained in $d_{1}$ be $\delta_{i}$. Take an interval of the given set containing $\delta_{i}$ and denote it by $d_{2}$; and so on. We thus get a countable set of the given intervals, containing every internal point of the $\delta$ 's, and therefore of the given set, as internal points. On the other hand the $d$ 's being intervals of the given set, every point internal to them is internal to the given set. Thus the intervals $d_{1}, d_{2}, \ldots$ satisfy the requirements.

Q. E. D.

It may be remarked that the following has been incidentally proved:

Given any set of intervals, a countable set of intervals with the same internal points can be found, the end-points of these intervals being rational (or belonging to any convenient set dense everywhere).

The proof above given is considerably shorter than that originally given. The method too, as Dr. Bernstern points out, possesses the further advantage that it can be extended to prove the generalised theorem in which regions in space of any number of dimensions take the place of the intervals. It is only necessary to take, instead of intervals, triangles in the plane, or $(n+1)$-hedra in space of $n$-dimensions. By.induction it follows that the number of such triangles, or $(n+r)$-hedra, with rational vertices is countable, and the whole argument applies.

This extended theorem was enunciated in my paper on Regions and Sets of Regions ${ }^{*}$ ); the proof there given for the plane, with an indication of its extensability to higher space, was of the same character as the proof given below, but not so concise.

\section{Alternative proof of the above Theorem.}

Assume first that the set of intervals lies in a finite fundamental segment.

Let $e_{1}, e_{2}, \ldots$ be a sequence of constantly decreasing positive quantities, having zero as limit.

Take any one of the intervals, and let its length be greater than $e_{i}$.

If there is another of the intervals having a part of length greater than $e_{i}$ external to the first interval, we take this as second interval; and so on.

Determine $n$ so that the length of the fundamental segment is less than $n e_{i}$, then there cannot be as many as $n$ non-overlapping intervals of length greater than $e_{i}$ in the fundamental segment. We must then, after a finite number $\left(<n^{*}\right)$ of the intervals 
have been determined in the manner indicated, find that there are no more to be obtained. We then go on to $e_{i+1}, e_{i+2}$, and so on.

When we have exhausted the sequence of $e$ 's, we shall have determined a countable set of the given intervals, since corresponding to each $e$ there are a finite number only. Let these be $d_{1}, d_{2}, \ldots$.

If $P$ be a point which is not internal to at least one of the $d$ 's, there is, by the principle of the determination, no value of $r$ such that we can draw an interval of length $e_{r}$ with $P$ as middle point, lying entirely in one of the given intervals. Thus $P$ is not internal to any one of the given intervals. On the other hand, the $d$ 's being intervals of the given set, every point internal to them is internal to the given set. Thus the intervals $d_{1}, d_{2}, \ldots$ satisfy the requirements.

If the fundamental segment is infinite, it can be divided into a countable number of finite segments, in each of which the theorem holds. Thus a countable number of countable sets of the intervals suffice to cover every internal point. But a countable number of countable sets contain only a countable number of intervals. Thus the theorem is true in the infinite, as in the finite fundamental segment.

Q. E. D.

The following is an alternative proof of the generalised Heine-Borel Theorem, following immediately from the theorem just proved.

The generalised HeIne-Borel Theorem. - Given a set of intervals containing as internal points all the points of a closed set $G$, a finite number of the intervals can be chosen, so as to enclose every point of $G$.

For, by the preceding theorem, a countable set of the given intervals may be found having the same internal points as the given set. Take these in countable order and omit any which has not as internal point at least one point of $G$ not internal to one of the preceding intervals. Let the intervals so determined be $d_{1}, d_{2}, \ldots$ and let $P_{i}$ be a point of $G$ not contained in $d_{1}, d_{2}, \ldots d_{i-1}$.

Then, if there are a countably infinite number of the points $P_{i}$, they have at least one limiting point $L$, which cannot be internal to $d_{\mathrm{r}}$, since at most two of the points $P_{2}, P_{3}, \ldots$ can be end-points of $d_{\mathrm{r}}$, and the rest are external to $d_{\mathrm{r}}$, and cannot therefore have a limiting point internal to $d_{\mathrm{t}}$. Similarly $L$ would not be internal to $d_{i}$ for any value of $i$, and therefore $L$ could not be a point of $G$, which is impossible since $G$ is closed. Thus the points $P_{1}, P_{2}, \ldots$ are finite in number, and the same is therefore true of the intervals $d_{1}, d_{2}, \ldots$ which contain every point of $G$.

Q. E. D.

Göttingen, November, 1905.

W. H. Youn G. 